\title{
Familial hypomagnesaemia with hypercalciuria and nephrocalcinosis maps to chromosome $3 q 27$ and is associated with mutations in the PCLN-1 gene
}

\author{
Stefanie Weber ${ }^{1}$, Katrin Hoffmann ${ }^{2}$, Nikola Jeck ${ }^{1}$, Kathrin Saar ${ }^{2}$, Martin Boeswald ${ }^{3}$, \\ Eberhard Kuwertz-Broeking ${ }^{4}$, Ivan IC Meij ${ }^{5}$, Nine VAM Knoers ${ }^{5}$, Pierre Cochat ${ }^{6}$, \\ Tereza Šuláková ${ }^{7}$, Klaus E Bonzel $^{8}$, Marianne Soergel ${ }^{1}$, Friedrich Manz ${ }^{9}$, Karl Schaerer $^{10}$, \\ Hannsjoerg W Seyberth ${ }^{1}$, André Reis ${ }^{2}$ and Martin Konrad ${ }^{1}$
}

\begin{abstract}
${ }^{1}$ Department of Pediatrics, Philipps University, Marburg; ${ }^{2}$ M ikrosatellitenzentrum, Max-D el brueck-Centre, Berlin; ${ }^{3}$ D epartment of Pediatrics, University Hospital, Erlangen; ${ }^{4}$ Department of Pediatrics, University Hospital, Münster, Germany; ${ }^{5}$ Department of Human Genetics, University Hospital Nijmegen, The Netherlands; ${ }^{6}$ Pediatric Nephrology Unit, Edouard-Herriot Hospital, Lyon, France; ${ }^{7}$ D epartment of Pediatrics, University Hospital, Ostrava, Czech Republic; ${ }^{8}$ D epartment of Pediatric Nephrology, University Children's Hospital, Essen; ${ }^{9}$ Research Institute of Child Nutrition, D ortmund; ${ }^{10}$ Department of Pediatric Nephrology, University Children's Hospital, Heidel berg, Germany
\end{abstract}

Familial hypomagnesaemia with hypercalciuria and nephrocalcinosis (FHHNC, MIM 248250) is a complex renal tubular disorder characterised by hypomagnesaemia, hypercalciuria, advanced nephrocalcinosis, hyposthenuria and progressive renal failure. The mode of inheritance is autosomal recessive. A primary defect in the reabsorption of magnesium in the medullary thick ascending limb of the loop of Henle (mTAL) has been proposed to be essential in FHHNC pathophysiology. To identify the underlying genetic defect we performed linkage analysis in eight families, including three with consanguineous marriages. We found linkage to microsatellite markers on chromosome $3 q 27$ with a maximum two-point lod score $\left(Z_{\max }\right)$ of 5.208 for D3S3530 without evidence for genetic heterogeneity. Haplotype analysis revealed crucial recombination events reducing the critical interval to $6.6 \mathrm{cM}$. Recently, mutations in the gene PCLN-1, mapping to 3q27 and coding for paracellin-1, were identified by Simon et al (1999) as the underlying genetic defect in FHHNC. Paracellin-1 represents a renal tight junction protein predominantly expressed in the TAL. Mutational analysis in our patient cohort revealed eight different mutations in the PCLN-1 gene, within six novel mutations. In seven of 13 mutant alleles we detected a Leu151 substitution without evidence for a founder effect. Leu151 is a residue of the first extracellular loop of paracellin-1, the part of the protein expected to bridge the intercellular space and to be important for paracellular conductance. This study confirms the implication of paracellin-1 defects in FHHNC and points to a predominant role of this protein in the paracellular reabsorption of divalent cations in the TAL. European Journal of Human Genetics (2000) 8, 414-422.

Keywords: hypomagnesaemia; hypercalciuria; nephrocalcinosis; renal failure; paracellin-1; PCLN-1

\section{Introduction}

Familial hypomagnesaemia with hypercalciuria and nephrocalcinosis (FHHNC, MIM 248250), first described by Michelis

Correspondence: Martin Konrad, MD, Department of Pediatrics, Philipps University, Deutschhausstrasse 12, D-35037 Marburg, Germany. Tel: +496421 2862789; Fax: +496421 2868956; E-mail:

konradm@mailer.uni-marburg.de

Received 1 July 1999; revised 18 January 2000; accepted 21 January 2000 et al in 1972, is a rare inherited renal tubular disease frequently complicated by progressive renal failure during childhood or adolescence. ${ }^{1-6}$ The mode of inheritance is autosomal recessive. Besides marked hypomagnesaemia the affected individuals present with polyuria, hyposthenuria, hypercalciuria, advanced nephrocal cinosis, low citrate excretion and impaired glomerular filtration rate (GFR). In addition, nephrolithiasis, recurrent urinary tract infections and incomplete distal renal tubular acidosis (dRTA) have been 
described. ${ }^{2,7}$ Some authors report elevated serum PTH levels early during the course of disease, independent of reduced GFR and therefore probably reflecting chronic calcium depletion. ${ }^{6}$ Ocular abnormalities, such as corneal calcifications, chorioretinitis, horizontal nystagmus and severe myopia have been described as inconsistent findings in families affected by FHHNC. ${ }^{6,8}$ In family members not affected by FHHNC, history of hypercalciuria and kidney stones is frequent. $^{6}$

FHHNC has to be differentiated from other forms of familial hypomagnesaemia. The autosomal dominant form of isolated renal magnesium loss (MIM 154020) has recently been mapped to chromosome11q23 in two large Dutch families probably sharing a common ancestor. ${ }^{9}$ The localisation of the genetic defect of the autosomal recessive form (MIM 248250) is still unknown. In contrast to FHHNC urinary calcium excretion is not elevated in these disorders. Hypocalciuria is found in the autosomal dominant form, normocalciuria in the recessive form.

Furthermore, hypomagnesaemia is a leading symptom in Gitelman syndrome (MIM 263800) (also described as the hypocal ciuric variant of Bartter syndrome) which is caused by mutations in the thiazide-sensitive $\mathrm{NaCl}$ cotransporter gene (SLC12A3).$^{10}$ In contrast to FHHNC this tubulopathy is associated with marked hypokalaemic metabolic alkalosis. Nephrocalcinosis has never been observed in Gitelman syndrome patients. In classic Bartter syndrome (MIM 241200), caused by mutations in the chloride channel gene CLCNKB, more than one third of the patients present with hypomagnesaemia. ${ }^{11}$ These patients also have pronounced hypokalaemic al kal osis. Nephrocal cinosis is present in less than $15 \%$ of the patients.

Not only renal magnesium reabsorption failure but also impaired intestinal absorption may result in hypomagnesaemia, as seen, for example, in familial hypomagnesaemia with secondary hypocal caemia (HSH, MIM 602014). A gene locus for HSH has been mapped to chromosome9q12-22.2 in three large inbred Bedouin kindreds. ${ }^{12}$

For a long time, the pathophysiology of FHHNC has been unclear. There has been indirect evidence from clinical observation and clearance studies that the primary defect is related to impaired magnesium reabsorption in the medullary thick ascending limb of the loop of Henle (mTAL). ${ }^{5}$ This segment plays a predominant role in renal magnesium reabsorption with magnesium passing mainly by paracellular flux, driven by the electrochemical gradient over the tubular epithelium. ${ }^{13}$ The hypermagnesiuria in FHHNC is associated with high renal calcium excretion rates. Hypercalciuria together with incomplete tubular acidosis resulting from hypomagnesaemia is accused to accelerate nephrocalcinosis and to enhance nephrolithiasis. Reduced concentrating abilities, recurring urinary tract infections and the development of renal insufficiency are regarded as a consequence of nephrocalcinosis. ${ }^{2}$ After exclusion of promising candidate genes known to be involved in tubular fluid and electrolyte transport we have performed a gen ome-wide linkage analysis with a cohort of eight families to localise the gene responsible for the disease and found linkage to chromosome3q27. Recently, the gene PCLN-1 coding for paracellin-1, a new member of the claudin family, has been cloned and mapped to this chromosomal region. ${ }^{14}$ PCLN-1 consists of 5 exons and encodes a protein of 305 amino acids length with four transmembrane domains and intracellular $\mathrm{NH}_{2}$ and $\mathrm{COOH}$ termini as predicted by sequence and hydrophilicity analysis. Further studies demonstrated colocalisation of paracellin-1 with the ubiquitous tight junction protein occludin. ${ }^{14}$ Due to these studies, paracellin-1 is regarded as a component of the tight junction complex. Expression of paracellin-1 was exclusively observed in the TAL and the distal convoluted tubule (DCT). Subsequently, mutations in PCLN-1 were identified in FHHNC. Ten different mutations in the PCLN-1 gene were described in a cohort of ten FHHNC families. ${ }^{14}$

We now report the identification of eight PCLN-1 mutations in our cohort of eight FHHNC families. Six of these are novel mutations of the PCLN-1 gene.

\section{Patients and methods Patients}

The study cohort consisted of eight FHHNC families of different ethnic origin with either two affected individuals and/or parental consanguinity (except F6) including 13 FHHNC patients (Figure1). The clinical course of two affected sisters (8054 and 8055) of the index family F1 has been reported previously. ${ }^{2}$ In the remaining patients, diagnosis was based on the following mandatory clinical symptoms: hypomagnesaemia $(<0.6 \mathrm{mmol} / \mathrm{l})$, hypercalciuria, bilateral nephrocal cinosis, polydipsia/polyuria, impai red glomerular filtration rate and the absence of hypokalaemic metabolic alkalosis. Additional findings were as follows: hypermagnesiuria was found in $11 / 11$ patients analysed, incomplete dRTA in 5/6, history of urinary tract infection in $7 / 12$, hypocitraturia in $9 / 9$ and elevated PTH levels in $7 / 9$. Three out of 12 patients had minor ocular abnormalities: convergent strabismus in one patient (8003 in F3) and astigmatism in two patients (8067 and 8068 in F7).

This study was approved by the local ethics committee and informed consent was obtained from the patients and/or their parents.

\section{Analysis of candidate gene loci}

Haplotype analysis was performed as described previously ${ }^{15}$ using flanking microsatellite markers which were linked to $11 q 23,{ }^{9}$ 9q12-9q22.2, ${ }^{12} \mathrm{KCNJ1},{ }^{16}$ SLC12A1, ${ }^{17}$ CLCNKA/ $\mathrm{CLCNKB},{ }^{18}$ and $\mathrm{KCC} 1 .{ }^{19}$

\section{Genome-wide linkage analysis}

Affected individuals and all available unaffected family members were genotyped in a genome-wide linkage analysis. A total of 180 microsatellites was typed until linkage was 


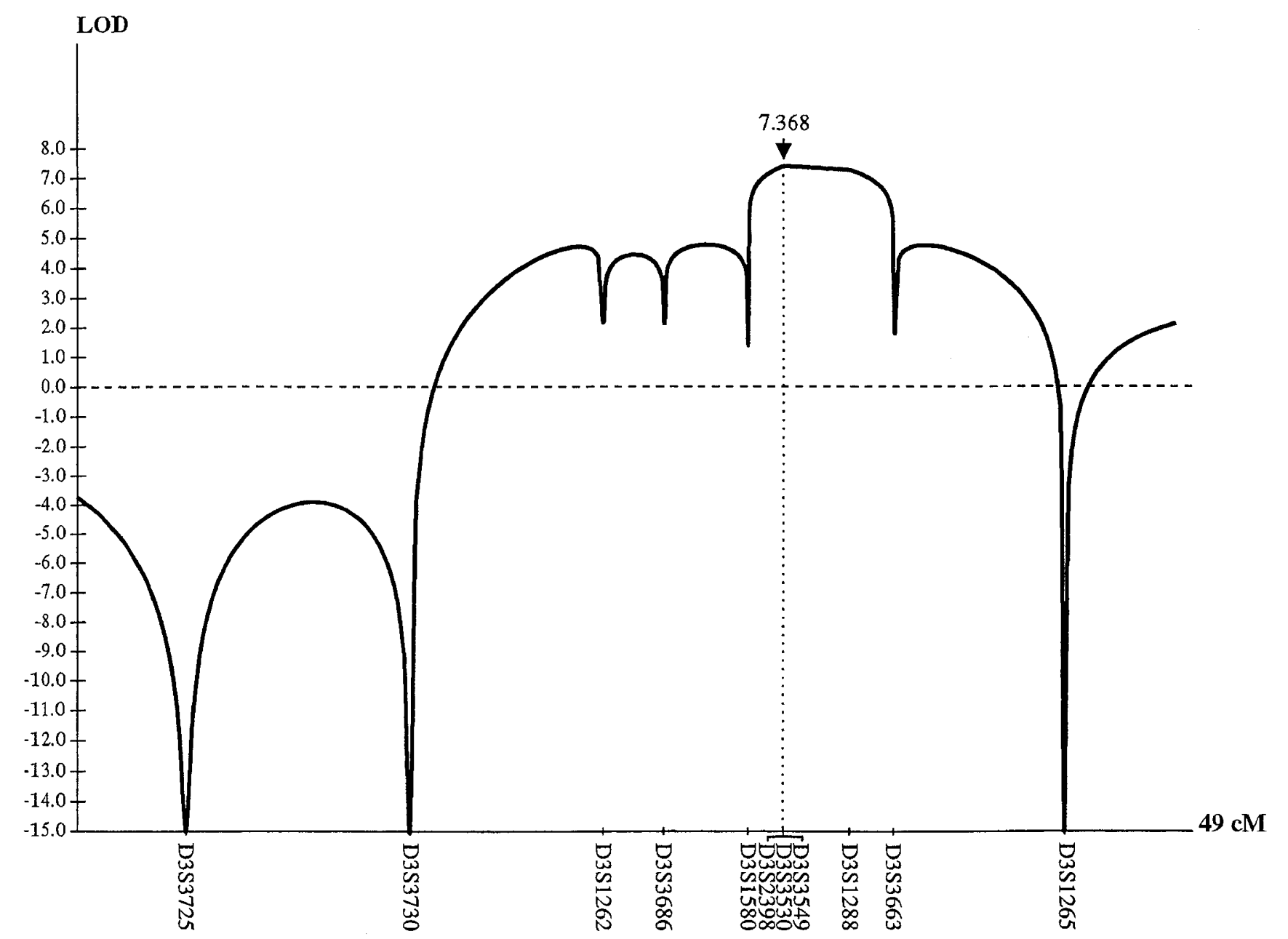

Figure 1 Multipoint analysis. Multipoint lod scores are given for a 49 cM on 3q26-28. Maximum lod score of 7.368 was obtained at the cluster D3S2398, D3S3530, D3S3549.

observed. Semi-automated genotyping was performed on ABI 377 DNA sequencers ( $A B I$, Foster City, CA, USA) as described. ${ }^{20}$ Data were analysed using the Genescan 2.1 and Genotyper 2.0 software (Perkin Elmer, Norwalk, CT, USA).

Two-point Lod-score calculations were performed by the LINKAGE program package ${ }^{21}$ using the LINKRUN computer program (TF Wienker, personal communication, 1999) under the hypothesis of an autosomal recessive mode of inheritance with $100 \%$ penetrance. Disease allele frequency was set at 0.0001 . For computation of multipoint lod scores, the program Genehunter version 2.0 was used. ${ }^{22}$ Two-point and multipoint analyses assumed equal allele frequencies. Haplotyping was performed also with Genehunter and by hand. The genetic maps and marker data were obtained from the 1996 Généthon map. ${ }^{23}$

\section{Mutational analysis}

After PCLN-1 had been identified as the gene responsible for FHHNC we examined the PCLN-1 gene in our cohort by single strand conformation polymorphism analysis (SSCA). Based on the sequence for the human PCLN-1 gene, an overlapping set of primers ${ }^{14}$ was used to amplify the coding sequence (exon 1-5) from genomic DNA by PCR. Amplified products were separated on polyacrylamide gels by electrophoresis (Pharmacia Biotech, Freiburg, Germany). Exons with conformational variants were directly sequenced using corresponding sequencing primers (ALF express sequencer, Pharmacia Biotech, Freiburg, Germany).

\section{Results}

\section{Exclusion of candidate genes and disease susceptibility} loci on $11 q$ and $9 q$

The loci on chromosome $11 q$ and $9 q$ which encompass the genes involved in isolated renal magnesium loss and $\mathrm{HSH}$, respectively, were excluded (data not shown) by testing flanking microsatellite markers reported in the original articles. $^{9,12}$ We also excluded by haplotype analysis several genes coding for proteins involved in tubular fluid and 
electrolytetransport, especially in the MTAL: the furosemidesensitive $\mathrm{NaK} 2 \mathrm{Cl}$ cotransporter (SLC12A1), the luminal potassium channel ROMK (KCNJ1), the basolateral chloride channels CLC-Ka and Kb (CLCNKA/B) and the basolateral $\mathrm{KCl}$ cotransporter (KCC1).

\section{Genome-wide linkage analysis}

Subsequently, a total genome scan was performed. Initial linkage was obtained for marker D3S3663 (3q27) in all eight families tested, with a lod score $Z=2.626$ at a recombination fraction $\Theta=0.05$. All families showed evidence for linkage to this region. Characterisation of additional markers revealed a maximum two-point lod score $\left(Z_{\max }\right)$ of 5.208 for D3S3530 at a recombination fraction $\Theta=0.00$, whereas multipoint analysis gave a $Z_{\max }$ of 7.368 around marker D3S2398 (Figure 1). In order to determine the exact location of the disease susceptibility locus haplotypes were constructed (Figure2). Heterozygosity values calculated from haplotype analysis data of familiesF1-F8 are given in Table1. Various phaseknown recombination events defined a $30 \mathrm{cM}$ interval

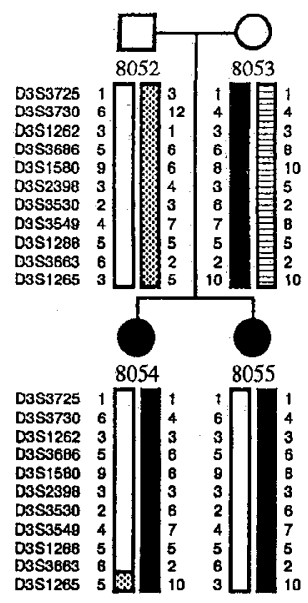

F1

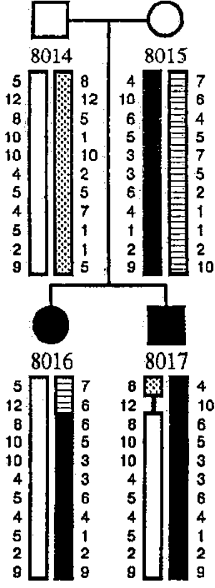

F2

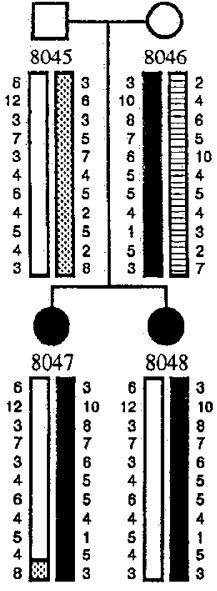

F5

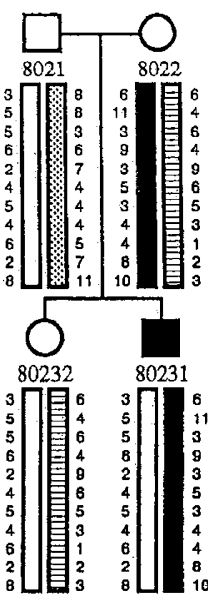

F6

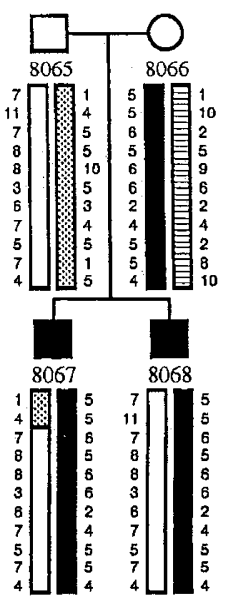

F7

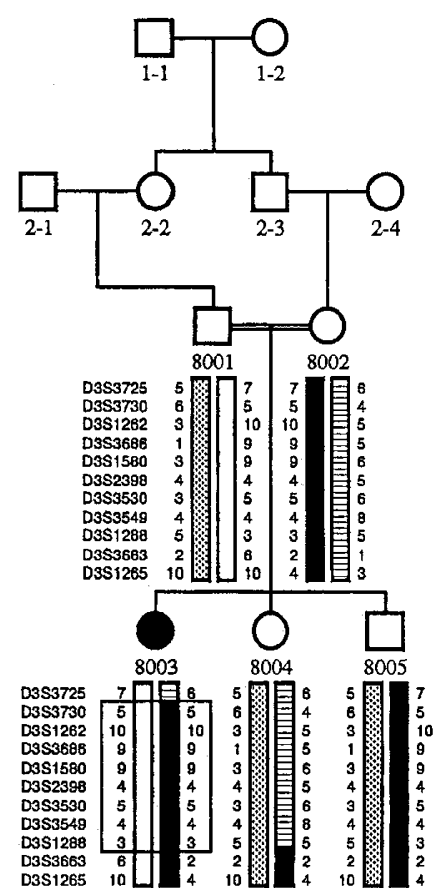

F3

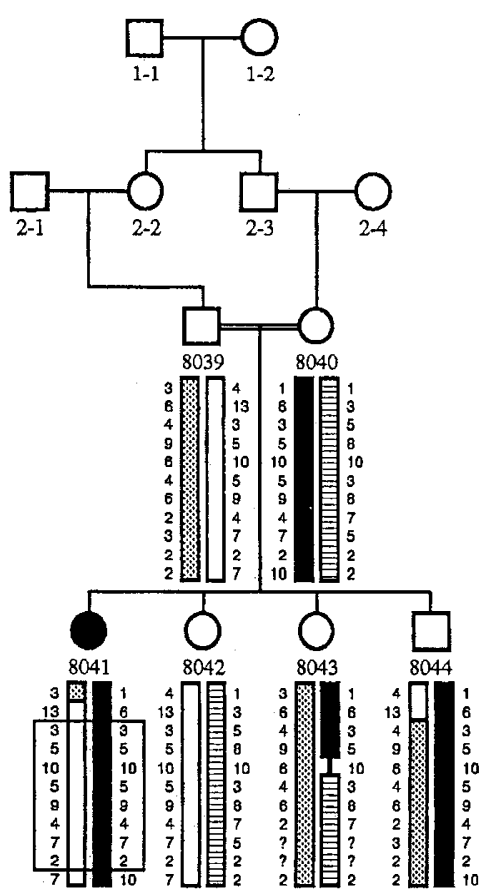

F4

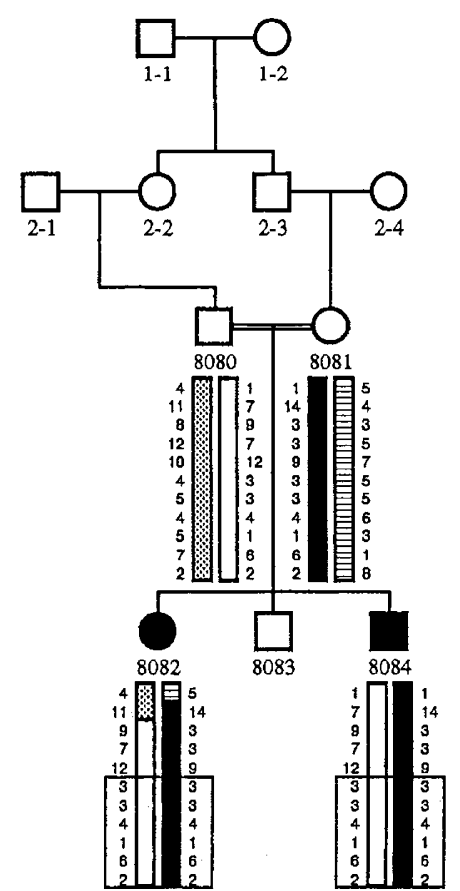

F8

Figure 2 Pedigrees and most likely marker haplotypes on chromosome 3q26-28 of FHHNC families investigated. Regions of homozygosity are framed. 
Table 1 Heterozygosity values of relevant markers on chromosome 3q26-28

\begin{tabular}{ll}
\hline Marker & Heterozygosity \\
\hline D3S3725 & 0.82 \\
D3S3730 & 0.797 \\
D3S1262 & 0.82 \\
D3S3686 & 0.695 \\
D3S1580 & 0.805 \\
D3S2398 & 0.758 \\
D3S3530 & 0.727 \\
D3S3549 & 0.609 \\
D3S1288 & 0.539 \\
D3S3663 & 0.703 \\
D3S1265 & 0.828
\end{tabular}

Values calculated from haplotype analysis data of eight FHHNC families (F1-F8).

between D3S3730 and D3S1265 harbouring the gene. Ancestral recombination events in consanguineous families (F3, F8) allowed further refinement of the critical region, restricting the interval to $6.6 \mathrm{cM}$ (Figure3).

\section{Mutational analysis of PCLN-1}

Mutational analysis of PCLN-1 by SSCA and direct sequencing revealed eight different mutations in our cohort of eight families, among these six novel mutations (Table2). All mutations cosegregate with the phenotype and none of these were found in 100 control chromosomes. Affected amino acid residues in the paracellin-1 protein are depicted in Figure4.

In two consanguinous but unrelated families of different ethnic origin (F4, F8) we identified a homozygous frameshift mutation, Arg55fs, affecting the $\mathrm{N}$-terminal part of paracellin-1. This early frameshift mutation results in truncation of the protein prior to the first transmembrane domain as it induces a nonsense amino acid sequence from amino acid position 55 to 89 followed by a TGA-stop codon at position 90. In F8, a second homozygous mutation on the same allele, His141Asp, was found in addition to the Arg55fs. Since the His141Asp exchange is located further downstream to the Arg55fs, we consider it to be nonfunctional, at least in this special case.

In the index family F1 (referring to the clinical description of the disease ${ }^{2}$ ) sequence analysis revealed a Leu151Phe exchange combined with a heterozygous preterm stop codon in exon 2 (Trp117X). This preterm stop results in deletion of the terminal 188 amino acids, truncating the protein between the two extracellular loops.

Interestingly, the amino acid position Leu151 was frequently mutated in our patients. Seven of 13 mutant alleles (F1, F2, F5, F6, F7) displayed a point mutation at this residue. A heterozygous Leu151Phe exchange is combined with a heterozygous Leu151Trp exchange in F2, with a heterozygous Leu145Pro exchange in F5 and with a heterozygous Gly239Arg exchange in F6. In the affected patients of F7 sequence analysis revealed a homozygous Leu151Phe substitution despite unrelated parents. Unknown consanguinity in this family could largely be ruled out by haplotype analysis.

The accumulation of Leu151Phe substitutions in our cohort raises the question of a founder effect. However, a common haplotype did not significantly emerge from haplotype analysis of the PCLN-1 flanking markers.

Beside these six novel molecular variants of the PCLN-1 gene we found a Gly198Asp (F3) and a Gly239Arg substitution in our cohort (F6) which have also been described by Simon et al in two Spanish kindreds and two Ital ian kindreds, respectively. ${ }^{14}$

\section{Discussion}

Until recently, little has been known about renal magnesium tran sport systems (for review see Quamme). ${ }^{13}$ Only 5-15\% of the ultrafiltrated magnesium are reabsorbed in the proximal tubule, while $50-60 \%$ are reabsorbed in the loop of Henle. Another $10 \%$ (corresponding to $80 \%$ of total magnesium delivered to this segment) are reabsorbed in the distal tubule.

Electrophysiological studies indicate that the majority of the magnesium absorption in the loop of Henle is paracellular, depending on electrochemical ion gradients and the transmembrane voltage. ${ }^{24,25}$ In addition, it has been shown in rats that magnesium also enters the cortical TAL cells via selective pathways, most likely through magnesium specific channels. ${ }^{26}$ Magnesium transporters have only been reported in bacteria and yeast. ${ }^{27-29}$ As the primary defect in FHHNC has been related to a reabsorption defect of magnesium in the TAL, investigation of the molecular aspects of FHHNC seemed promising in order to gain more insight into renal magnesium transport systems. In the present study we have mapped a gene locus responsible for FHHNC to chromosome3q27. Refined mapping has restricted the interval to a genetic distance of less than $6.6 \mathrm{cM}$ between the loci D3S1580 and D3S3663. Genetic heterogeneity seems unlikely since haplotypes of all families in our study were compatible with linkage to this region. Using the same approach with an independent cohort of 12 FHHNC families Simon and colleagues were able to map the gene to a $1 \mathrm{cM}$ interval on $3 q 27$ and subsequently identified mutations in the PCLN-1 gene coding for paracellin-1 as the underlying defect in FHHNC in 10 families.

We identified homozygous or compound heterozygous mutations in all affected individuals of our FHHNC patient cohort, supporting the hypothesis of FHHNC being a monogenetic disease. Interestingly, mutational analysis revealed a striking predominance of mutations affecting the Leu151 residue (seven of 13 mutant alleles). Especially the accumulation of Leu151Phe substitutions in our patient cohort (F1, F2, F5, F6, F7) might give rise to the proposal of a founder effect but haplotype studies did not point to a common ancestor in 


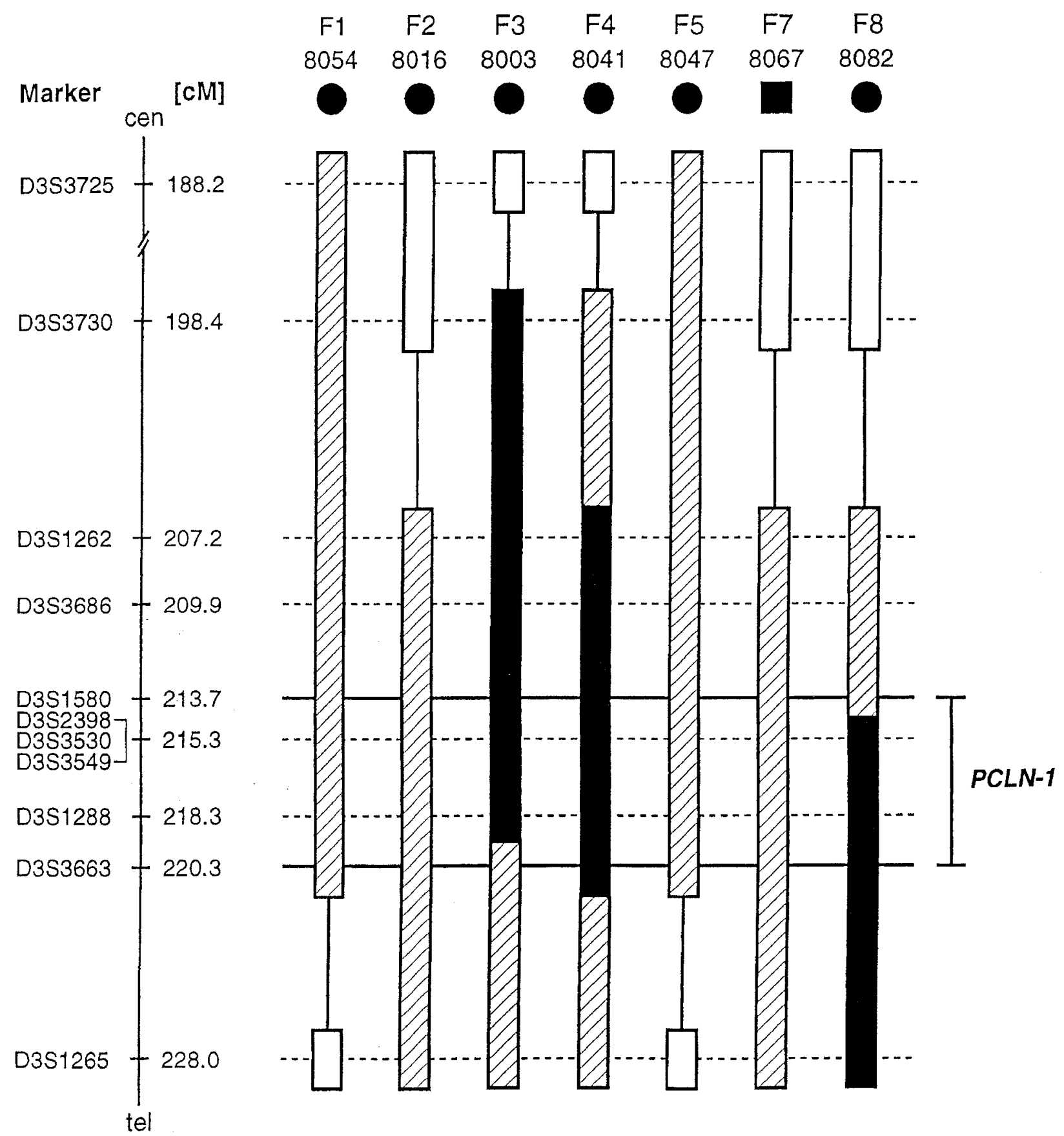

Figure 3 Key recombination events in FHHNC families investigated. Selected haplotypes from Figure 2 are given for chromosomes with recombination events refining the localization of the FHHNC gene locus. Wild type haplotypes are in open boxes, mutant haplotypes are hatched and homozygous segments in families of consanguineous marriages (F3, F4 and F8) are solid. Ancestral recombination events between markers D3S1580 and D3S2398 in family F8 and between D3S1288 and D3S3663 in family F3 define the proximal and distal borders of a $6.6 \mathrm{cM}$ segment harbouring the gene locus. Marker coordinates are taken from the final Généthon linkage map. ${ }^{23}$

these families. Therefore, these mutations appear to have occurred independently. However, it has been demonstrated that haplotype analysis cannot completely rule out a founder effect: even in a homogenous population, significant disequilibrium could not be demonstrated for loci located $900 \mathrm{~kb}$ and $1600 \mathrm{~kb}$ away from the disease gene. ${ }^{30}$ As our 
Table 2 Mutational analysis of PCLN-1 in eight FHHNC families (F1-F8)

\begin{tabular}{|c|c|c|c|c|c|c|}
\hline Family & Sex & Ethnic origin & Consanguinity & Zygosity & DNA change $\mathrm{e}^{\mathrm{a}}$ & Protein change \\
\hline F1-1/2 & $f / f$ & German & no & comp hetero & $350 \mathrm{G} \rightarrow \mathrm{A}-453 \mathrm{G} \rightarrow \mathrm{T}$ & Trp117X-Leu151Phe \\
\hline $\mathrm{F} 2-1 / 2$ & $\mathrm{f} / \mathrm{m}$ & German & no & comp hetero & $452 T \rightarrow G-453 G \rightarrow T$ & Leu151Trp-Leu151Phe \\
\hline F3 & $\mathrm{f}$ & Turkish & yes & homo & $593 \mathrm{G} \rightarrow \mathrm{C}$ & Gly198Asp \\
\hline F4 & $f$ & Tunisian & yes & homo & $165 / 166$ delGG $\rightarrow$ insC & Arg55fs \\
\hline F5- $1 / 2$ & $f / f$ & German & no & comp hetero & $434 \mathrm{~T} \rightarrow \mathrm{C}-453 \mathrm{G} \rightarrow \mathrm{T}$ & Leu145Pro-Leu151Phe \\
\hline & $\mathrm{m}$ & Bosnian & no & comp hetero & $453 \mathrm{G} \rightarrow \mathrm{T}-715 \mathrm{G} \rightarrow \mathrm{A}$ & Leu151Pro-Gly239Arg \\
\hline F7-1/2 & $\mathrm{m} / \mathrm{m}$ & Czech & no & homo & $453 \mathrm{G} \rightarrow \mathrm{T}$ & Leu151Phe \\
\hline F8- $1 / 2$ & $\mathrm{f} / \mathrm{m}$ & Tunisian & yes & homo-homo & $165 / 166 \mathrm{delGG} \rightarrow$ insC $-421 \mathrm{C} \rightarrow \mathrm{G}$ & Arg55fs-His141Asp ${ }^{b}$ \\
\hline
\end{tabular}

f: female; m: male; comp hetero: compound heterozygous; homo: homozygous; del: deletion; ins: insertion; fs: frameshift; trp: tryptophane; leu: leucine; phe: phenylalanine; gly: glycine; asp: asparagine; arg: arginine; pro: proline; his: histidine. ${ }^{a} \mathrm{DNA}$ nucleotid position count is based on ATG (start-methionine) as first position of PCLN-1 cDNA (gbAF152101). ${ }^{\text {}}$ nonfunctional due to homozygous Arg55fs.

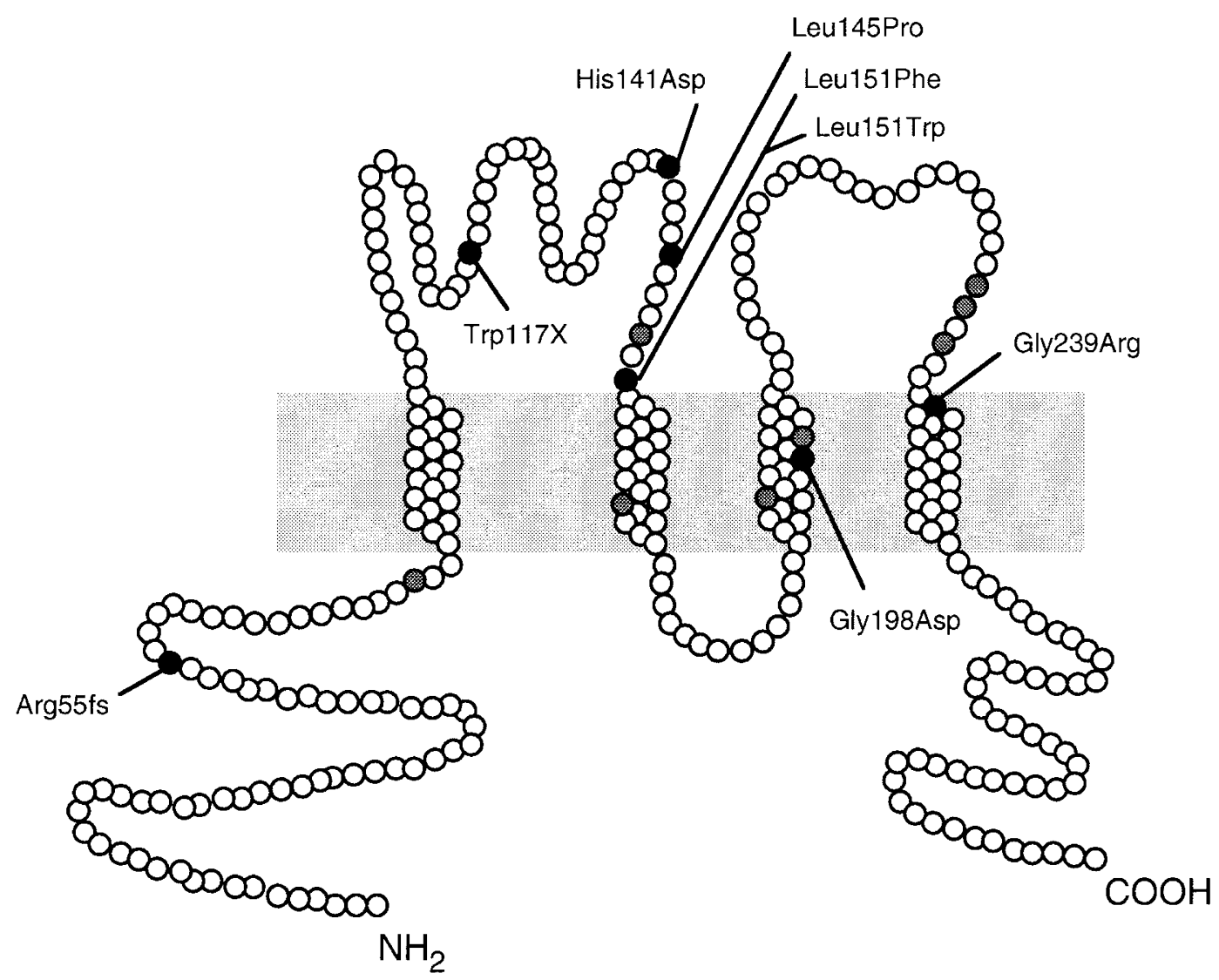

Figure 4 Paracellin-1 protein model deduced from hydrophilicity plots. ${ }^{14}$ Amino acid residues affected by PCLN - 1 mutations are shaded (mutations previously described $\mathrm{in}^{14}$ ) and solid (mutations analysed in familiesF1-F8 of this study). The Gly198Asp and Gly239Arg substitutions have also been described by Simon et al. ${ }^{14}$

cohort is still too small to support or completely rule out this theory, further analysis of the PCLN-1 gene in a larger cohort of FHHNC families will be necessary.

Assuming an independent occurrence of the recurring amino acid substitutions the existence of mutational hot spots in PCLN-1 seems possible. Leu151 is located in the first extracellular loop of paracellin-1. This part of the protein is thought to bridge the intercellular space and probably participates in the paracellular conductance of divalent cations, especially of magnesium ions. Due to the frequency of mutations affecting Leu151 we propose this amino acid residue to be of high functional importance. Functional expression studies of mutated paracellin-1 protein will help to clarify the implication of the detected mutations in paracellin-1 function.

\section{Clinical implications}

As far as we know to date, loss or impairment of paracellin-1 function results in defective reabsorption of magnesium ions in the kidney leading to profound hypermagnesiuria and 
hypomagnesaemia. The defect in renal magnesium handling in FHHNC is associated with high excretion rates of calcium. This might be explained by the fact that calcium and magnesium transport systems in the kidney are frequently linked, which has also been demonstrated for the paracellular pathway of both divalent cations in the MTAL. ${ }^{13}$ However, serum calcium levels in FHHNC patients remain within the normal range. Hypocal caemia seems to be prevented by alternative pathways in calcium homeostasis: transcellular tubular calcium reabsorption in the DCT, intestinal calcium absorption and calcium release out of the bone substance, mediated by different hormones such as 1,25- $(\mathrm{OH})_{2}$-cholecal ciferol and parathyroid hormone.

Despite normocalcaemia, hypercalciuria seems to play an important role in FHHNC pathophysiology. Interestingly, Praga et al discovered a striking incidence of hypercal ciuria in healthy family members of FHHNC patients. Eleven of 26 (42\%) examined individuals had hypercalciuria without nephrocalcinosis or renal insufficiency, but two presented with mild hypomagnesaemia and four with recurrent nephrolithiasis. It was speculated that isolated hypercalciuria might appear as a milder phen otype of the disease, consistent with an autosomal dominant mode of inheritance with variable phenotypic expression. ${ }^{6}$ Accepting an autosomal recessive mode of inheritance of FHHNC, as supported by the results of linkage and mutational analysis, family members with hypercalciuria and/or nephrolithiasis seem to be symptomatic heterozygotes - a phenomenon not commonly seen in autosomal recessive diseases.

In our cohort of eight FHHNC families nephrolithiasis/ hypercalciuria was observed in three parents (F1, F3, F5). Since the mutant allele frequency can be expected to be low in the common population, it would be interesting to test families with isolated hypercalciuria/nephrolithiasis with apparently autosomal dominant mode of transmission for cosegregation with the PCLN-1 locus and mutations in the PCLN-1 gene.

Furthermore, in two family members of FHHNC patients described 1995 by Praga et al who were not affected by FHHNC but presented with hypercalciuria and nephrolithiasis, intravenous urography revealed the characteristic radiological picture of medullary sponge kidneys (MSK). ${ }^{6}$ An association of FHHNC and MSK has also been reported previously in two brothers. ${ }^{31}$ In a German FHHNC patient of a non-informative family (therefore not included in this study), renal sonography did not only show bilateral nephrocalcinosis but also multiple small renal cysts and diagnosis of MSK was proposed (personal communication, J Missel witz, 1997). The mother of this patient suffers from nephrolithiasis. To date, a distinct familial transmission of MSK has not been established. Since MSK patients frequently present with hypercalciuria and nephrolithiasis, ${ }^{32}$ closer investigation of the coincidence of FHHNC and MSK would be promising.

In conclusion, our study supports the theory of FHHNC to be a monogenetic disease, associated with mutations in the
PCLN-1 gene. The responsible gene locus maps to chromosome3q27. As the primary defect in FHHNC is related to a dysfunction of a renal tight junction protein, FHHNC can be considered as a hereditary tight junction disease. Additional studies of paracellin-1 function in the tight junction complex might elucidate further aspects of FHHNC pathophysiology.

\section{Acknowledgements}

The authors are indebted to the participating patients and their families for their cooperation. We would like to thank Franz Rüschendorf for multipoint linkage analysis, Thomas $F$ W ienker for the LINKRUN computer program, and Nora Alffen for excellent technical assistance.

MK and HWS were supported by the Deutsche Forschungsgemeinschaft (Ko1480/3-2). The Mikrosatellitenzentrum at the MDC is supported by a grant-in-aid from the German Human Genome Project to AR.

\section{References}

1 Michelis MF, Drash AL, Linarelli LG et al: Decreased bicarbonate threshold and renal magnesium wasting in a sibship with a distal tubular acidosis. Metabolism 1972; 21: 905-920.

2 Manz F, Schärer $K$, Janka $P$ et al: Renal magnesium wasting, incomplete tubular acidosis, hypercalciuria and nephrocal cinosis in siblings. Eur J Pediatr 1978; 128: 67-79.

3 EvansRA, Carter JN, George CR et al: The congenital “magnesiumlosing kidney". Report of two patients. Q J Med 1981; 50: 39-52.

4 UImann A, Hadj S, Lacour B et al: Renal magnesium and phosphate wastage in a patient with hypercalciuria and nephrocalcinosis: effect of oral phosphorus and magnesium supplements. Nephron 1985; 40: 83-87.

5 Rodriguez-Soriano J, Vallo A, Garcia-Fuentes M: Hypomagnesaemia of hereditary origin. Pediatr Nephrol 1987; 1: 465-472.

6 Praga M, Vara J, Gonzalez-Parra E et al: Familial hypomagnesemia with hypercalciuria and nephrocalcinosis. Kidney Int 1995; 47: 1419-1425.

7 Rodriguez-Soriano J, Vallo A: Pathophysiology of the renal acidification defect present in the syndrome of familial hypomagnesaemia-hypercalciuria. Pediatr Nephrol 1994; 8: 431-435.

8 Meier W, Blumberg A, Imahorn W et al: Idiopathic hypercalciuria with bilateral macular colobomata: a new variant of oculo-renal syndrome. Helv Paediat Acta 1979; 34: 257-269.

9 Meij IC, Saar K, van den Heuvel LPWJ et al: Hereditary isolated renal magnesium loss maps to chromosome11q23. Am J Hum Genet 1999; 64: 180-188.

10 Simon DB, Nelson-Williams C, Bia MJ et al: Gitelman's variant of Bartter syndrome, inherited hypokalemic alkalosis, is caused by mutations in the thiazide-sensitive $\mathrm{Na}-\mathrm{Cl}$ cotransporter. Nat Genet 1996; 12: 24-30.

11 Konrad $\mathrm{M}$, Vollmer $\mathrm{M}$, Lemmink $\mathrm{HH}$ et al: Mutations in the chloride channel gene, CLCNKB, cause Bartter syndrome. J Am Soc Nephrol 1998; 9: 390A.

12 Walder RY, Shalev H, Brennan TMH et al: Familial hypomagnesemia maps to chromosome $9 q$, not to the $X$ chromosome: genetic linkage mapping and analysis of a balanced translocation breakpoint. Hum Mol Genet 1997; 6: 1491-1497.

13 Quamme GA: Renal magnesium handling. New insights in understanding old problems. Kidney Int 1997; 52: 1180-1195.

14 Simon DB, Lu Y, Choate KA et al: Paracellin-1, a renal tight junction protein required for paracellular $\mathrm{Mg}^{2+}$ resorption. Science 1999; 285(5424): 103-106. 
15 International Collaborative Study Group for Bartter-Like Syndromes: Mutations in the gene encoding the inwardly-rectifying renal potassium channel, ROMK, cause the antenatal variant of Bartter syndrome: evidence for genetic heterogeneity. Hum Mol Genet 1997; 6: 17-26.

16 Simon DB, Karet FE, Rodriguez-Soriano J et al: Genetic heterogeneity of Bartter's syndrome revealed by mutations in the $\mathrm{K}^{+}$ channel, ROMK. Nat Genet 1996; 14: 152-156.

17 Simon DB, Karet FE, Hamdan JM et al: Bartter's syndrome, hypokalemic alkal osis with hypercal ciuria, is caused by mutations in the Na-K-2Cl cotransporter NKCC2. Nat Genet 1996; 13: 183-188.

18 Simon DB, Bindra RS, Mansfield TA et al: Mutations in the chloride channel gene, CLCNKB, cause Bartter's syndrome typeIII. Nat Genet 1997; 17: 171-178.

19 Larsen F, Solheim J, Kristensen $\mathrm{T}$ et al: A tight cluster of five unrelated human genes on chromosome16q22.1. Hum Mol Genet 1993; 2: 1589-1595.

20 Saar K, Chrzanowska KH, Stumm M et al: The gene for the ataxiateleangiectasia variant, Nijmegen breakage syndrome, maps to a 1-cM interval on chromosome8q21. Am J Hum Genet 1997; 60: 605-610.

21 Lathrop GM, Lalouel JM: Easy calculations of lod scores and genetic risks on small computers. Am J Hum Genet 1984; 36: 460-465.

22 Kruglyak L, Lander ES: Complete multipoint sib-pair analysis of qualitative and quantitative traits. Am J Hum Genet 1995; 57: 439-454.

23 Dib C, Fauré S, Fizames $C$ et al: A comprehensive genetic map of the human genome based on 5,264 microsatellites. Nature 1996; 380: 152-154.
24 Greger R: Ion transport mechanisms in thick ascending limb of Henle's loop of mammalian nephron. Physiol Rev 1985; 65: 760-797.

25 DeRouffignac C, Quamme GA: Renal magnesium handling and its hormonal control. Physiol Rev 1994; 74: 305-322.

26 Dai L-J, Quamme GA: Intracellular $\mathrm{Mg}^{2+}$ and magnesium depletion in isolated renal thick ascending limb cells. J Clin Invest 1991; 88: 1255-1264.

27 Smith DL, Maguire ME: Molecular aspects of $\mathrm{Mg}^{2+}$ transport systems. Miner Electrolyte M etab 1993; 19: 266-276.

28 Townsend DE, Esenwine AJ, George J III et al: Cloning of the mgtE $\mathrm{Mg}^{2+}$ transporter from Providencia stuartii and the distribution mgtE in gram-negative and gram-positive bacteria. J Bacteriol 1995; 177: 5350-5354.

29 MacDiarmid CW, Gardner RC: Overexpression of the Saccharomyces cerevisiae magnesium transport system confers resistance to aluminium ion. J Biol Chem 1998; 273: 1727-1732.

30 Hästbacka J, de la Chapelle A, Kaitila I et al: Linkage disequilibrium mapping in isolated founder populations: diastrophic dysplasia in Finland. Nat Genet 1992; 2: 204-211.

31 UImann A, Hadj S, Lacour B et al: Renal magnesium and phosphate wastage in a patient with hypercalciuria and nephrocalcinosis: effect of oral phosphorus and magnesium supplements. Nephron 1985; 40: 83-87.

32 Parks JH, Coe FL, Strauss AL: Calcium nephrolithiasis and medullary sponge kidney in woman. N Engl J Med 1982; 306: 1088-1091. 